\title{
Quantum-percolation model of electronic transport in two-dimensional granular metal films
}

\author{
Zhao-Qing Zhang* and Ping Sheng \\ Exxon Research and Engineering Company, Route 22 East, Annandale, New Jersey 08801
}

(Received 31 January 1991)

\begin{abstract}
We study the macroscopic and mesoscopic transport properties of granular metal films by using a quantum-percolation model. A granular metal film with metal fraction $p$ is simulated by conducting grains randomly occupying the sites of a square lattice with probability $p$. Electrons are allowed to hop between nearest-neighbor grains. This model incorporates the granular property of a film and enables us to study the magnetoconductance in both the weak- and strong-scattering regimes. The calculated magnetoconductance exhibits a sign change as the temperature varies, as well as oscillations as a function of the applied magnetic field. Both are consistent with some recent experimental data. The universal conductance fluctuations and resonant-tunneling characteristics are also studied systematically in both the extended and the localized regimes of mesoscopic samples. At the percolation threshold, the localization behavior is studied by using both the finite-size-scaling method and the resonant-tunneling method. Our results show no evidence for superlocalization. The effect of a magnetic field on localized wave functions at the percolation threshold is also examined.
\end{abstract}

\section{INTRODUCTION}

Granular metals are metal-insulator composites formed by cosputtering or coevaporation. They are mostly in the form of thin films $<1 \mu \mathrm{m}$ in thickness. Over the past two decades, the electrical transport properties of granular metal films have been a topic of active experimental and theoretical study. ${ }^{1}$ In the dielectric regime, where the metal grains are dispersed in an insulating matrix, electric conduction is by the hopping mechanism in which the charge carriers are transported from grain to grain via thermally activated tunneling., 2 In the metallic regime, on the other hand, electrons can percolate directly through connected metallic networks so that the conductivity is expected to exhibit metallic behavior. However, what we have actually observed show significant deviation from the usual metallic behavior. In particular, it was found that at low temperatures, i.e., $T<10 \mathrm{~K}$, all granular metal films exhibit the behavior of weakly increasing resistance as the temperature is decreased. ${ }^{4}$ Also, the low-temperature magnetoresistance was found to have the opposite sign as that of metal. These behaviors have been interpreted as the manifestations of electron localization effects. ${ }^{5,6}$ The outline of this interpretation is easy to appreciate and is briefly described below.

Since by definition a granular metal is a dirty metal, election-impurity scattering is inherent and important in these materials. A direct consequence of multiple electron-impurity scattering is the well-known diffusive transport behavior. In the past decade, however, it was found that wave diffusion differs from classical particle diffusion in one important aspect, i.e., the coherent backscattering effect. ${ }^{6}$ As a general wave phenomenon, what this effect states is that as long as all the scatterings are elastic and time reversal invariant, the scattered wave in the $180^{\circ}$ backward direction is always coherent and should therefore exhibit constructive interference. As a result, scattering in the backward direction is twice as probable as in the other directions and can thus lead to a diminished diffusion constant (since a wave has less change of moving forward than backward). This effect has indeed been seen experimentally for light scattering from random media. ${ }^{7}$

The magnitude of this coherent backscattering effect depends on several factors. It is proportional to the scattering strength of the impurities. When the scattering is strong (negative) correction to the diffusion constant is correspondingly large. When the correction becomes equal in magnitude to the classical diffusion constant, the electron (or the wave) would be localized. The effect also relies on the time-reversal invariance of the medium. Thus for a uniformly moving medium or a medium with an externally applied magnetic field the coherent backscattering effect can be reduced, which implies an increase in the conductivity. This is the source of the negative magnetoresistance in granular metals. In addition, the effect's magnitude is also dependent on the sample size. In general, for a $2 \mathrm{D}$ weak-scattering sample of size $L \times L$ the renormalized conductivity (which is proportional to the diffusion constant by the Einstein relation) is given by ${ }^{5,6}$

$$
\sigma=\sigma_{0}-\frac{e^{2}}{\hbar \pi^{2}} \ln \left[\frac{L}{l}\right] .
$$

Here $\sigma_{0}$ is the normal-metal conductivity, $l$ denotes the elastic-scattering, mean free path, $e$ the electronic charge, and $\hbar$ Planck's constant. The sample-size dependence can be directly translated into temperature and magnetic-field dependence through the following argument. At finite temperatures, an electron has an inelastic scattering time $\tau_{\text {in }}$ which is inversely proportional to a power of the temperature $T$ as $\tau_{\text {in }} \propto T^{-p}$, where $p \simeq 1-3$. 
Since the coherent backscattering effect relies on all the scatterings being elastic, its sample size may be defined by a dephasing length $L_{\phi} \propto \sqrt{D_{0} \tau_{\text {in }}}=\sqrt{l l_{\text {in }} / 2}$, where $D_{0}$ is the classical diffusion constant and $l_{\text {in }}=v \tau_{\text {in }}$ is defined as the inelastic scattering length. By using the dephasing length in Eq. (1), one immediately gets a $\delta \sigma \propto \ln T$ temperature dependence for the correction term. This is usually the explanation for the observed increasing resistance for decreasing $T$ in granular metals. In words, what it means is that as $T$ decreases, $L_{\phi}$ increases, which implies a larger coherent backscattering effect and thereby a smaller diffusion constant. In similar manner, the application of a magnetic field creates a magnetic length $L_{H}=\sqrt{h c / 4 e H}$, where $c$ denotes the speed of light. When $L_{H}$ becomes smaller than the dephasing length $L_{\phi}$, $L_{H}$ becomes the relevant sample size in Eq. (1). That means

$$
\delta \sigma(H)=\frac{e^{2}}{2 \pi^{2} \hbar} \ln \left(\frac{H}{H_{0}}\right),
$$

where $H_{0}=h c / 4 e L_{\phi}^{2}$ is the characteristic magnetic-field strength at which $L_{H}=L_{\phi}$.

While the localization theory can give qualitative explanations to the observed transport phenomenon in granular metals, yet all the quantitative theories to data have been based on the assumptions of weak scattering and weak magnetic field. In granular metals, as the volume fraction of insulator increases, the validity of such weak-scattering assumption becomes increasingly dubious. Thus, extending the theory into the strong scattering and strong field regimes is one motivation for this work. Another important motivation is to study the mesoscopic transport behavior in 2D granular metal films. Mesoscopic is a term describing the material properties on a scale intermediate between the molecular and the bulk. Here it denotes the electrical conduction behavior at a sample size $L<L_{\phi}$. The important distinction of this transport regime is that all the scatterings are elastic so that there can be effects due to phase interference of the electronic wave functions. Universal conductance fluctuations is an example of such an effect ${ }^{8}$ recently found in mesoscopic conducting samples. In granular metals, the ability to continuously vary the amount of geometric randomness offers a new angle to study such phase interference effects.

In this work a granular metal film is simulated by a $2 \mathrm{D}$ quantum percolation model in which the sites of a simple square lattice are randomly occupied by metallic grains with a probability $p$, where $p \geq p_{c}=0.593$ to ensure the connectivity of the metallic network. Electrons are allowed to hop only between nearest-neighbor grains. The empty sites are regarded as insulating. Thus the randomness of the model arises purely from its geometry. By using Green's function method and the multichannel Landauer formula, ${ }^{9}$ the conductance of a finite sized model may be explicitly evaluated. For macroscopic conductance the calculated conductance is averaged over many different configurations. Our results indicate deviations from the weak-coupling predictions of Eq. (1) as well as a sign change in the magnetoresistance as temperature is varied. As a function of the magnetic field, the magnetoresistance also exhibits weak oscillations when the magnetic length becomes comparable to the granular particle. These results are consistent with some recent experimental data. ${ }^{4}$ The properties of Anderson localization transition of this quantum percolation model has been studied by series expansion ${ }^{10}$ including the magnetic field effect. ${ }^{11}$

For mesoscopic conductance, on the other hand, it is the single configuration result that is important. Since the configurations are random, the object of our study is therefore the distribution of the conductances. We find three regimes. In the ballistic and weakly scattering transport regimes, i.e., sample size $L \ll$ localization length $\xi_{\text {loc }}$, the distribution is normal, and the rootmean-square conductance fluctuation is larger than $e^{2} / h$. In the very localized regime, i.e, $L \gg$ localization length, the distribution is log-normal and the conductance fluctuation is small. Between these two limits there is a transition regime in which the distribution of conductances is between normal and log-normal. For $10 l<L<\xi_{\text {loc }}$, i.e., in the transition regime and part of the normaldistribution regime, the root-mean-square fluctuation of the conductances is constant as a function of $L$ and on the order of $e^{2} / h$. By definition, this is the regime of universal conductance fluctuations. Our results therefore suggest experiments on granular metal films to verify the existence of the three distinct regimes by looking at conductance fluctuations and their variation with temperature, magnetic field, and $p$.

In what follows, Sec. II describes the quantum percolation model and the method of calculation. Results on macroscopic conductance and comparison with experiments is presented in Sec. III. Mesoscopic conductance behavior is presented in Sec. IV, followed by concluding remarks in Sec. $\mathrm{V}$.

\section{MODEL DESCRIPTION AND THE CALCULATIONAL APPROACH}

Consider a simple square lattice in which a fraction $p$ of the sites is occupied by metal grains. We will only consider the regime $p>p_{c}=0.593$ so that the metal grains always form a connected network. In the presence of a perpendicular magnetic field the Hamiltonian of the quantum percolation model can be written in the following tight-binding form:

$\boldsymbol{H}=\sum_{i} \varepsilon_{i}|i\rangle\left\langle i\left|+t \sum_{[i j]} \exp \left(-\frac{2 \pi i}{\phi_{0}} \int_{i}^{j} \mathbf{A} \cdot d \boldsymbol{l}\right)\right| j\right\rangle\langle i|$,

with

$$
\epsilon_{i}=\left\{\begin{array}{l}
0 \text { if site } i \text { is occupied by a metal particle } \\
\infty \text { of site } i \text { is occupied by the insulator }
\end{array}\right.
$$

where $\phi_{0}$ denotes the unit of quantum flux, $h c / e, \mathbf{A}$ is the vector potential, $t$ is the nearest-neighbor hopping matrix element, taken to be unity in this work, and $[i, j]$ denotes the nearest-neighbor site indices of a square lattice with a lattice constant $a$ taken to be the typical granular particle 
size $\sim 100 \AA$. In our numerical calculations the length is calibrated in units of $a$. In Eq. (3) the first term is the site energy term. Here we assign infinite site energy to insulators, which would prevent electrons from hopping to such sites. The second term describes nearest-neighbor metal grains. Here the applied magnetic field can introduce a position-dependent phase to the hopping matrix element, obtained by using the "Peierls substitution.", 12 This model can be easily generalized to models of finite thicknesses, so that 3D effects may be considered.

In order to calculate the conductance we connect two sides of a sample of size $L \times L$ by perfect leads, i.e., $p=1$. The conductance is evaluated by using the multichannel Landauer formula, ${ }^{9}$ i.e.,

$$
g=\frac{G}{\left(e^{2} / h\right)}=\frac{4\left(\sum_{i=1}^{L_{0}} T_{i}\right]\left[\sum_{i=1}^{L_{0}} v_{i}^{-1}\right]}{\sum_{i=1}^{L_{0}}\left(1+R_{i}-T_{i}\right) v_{i}^{-1}},
$$

where $L_{0}$ denotes the number of propagating channels in the leads. Hard wall boundary condition is chosen along the transverse direction $y$ with discrete transverse momentum index $k_{y}=\pi n /(L+2)(n=1,2, \ldots, L+1)$. The real solutions of $k_{x}$ in the following dispersion relation determine the number of allowed channels $L_{0}$ :

$$
\frac{E}{t}=2\left[\cos \left(k_{x} a\right)+\cos \left(k_{y} a\right)\right] \text {. }
$$

A factor of 2 due to spins has been included in Eq. (4). The values of $T_{i}$ and $R_{i}$ are related to the transmission and reflection matrices by

$$
\begin{aligned}
& T_{i}=\sum_{j=1}^{L_{0}}\left|t_{i j}\right|^{2}, \\
& R_{i}=\sum_{j=1}^{L_{0}}\left|r_{i j}\right|^{2},
\end{aligned}
$$

which in turn are calculated numerically by using recursive Green's function technique, ${ }^{13,14}$ with

$$
\left|t_{i j}\right|^{2}=v_{i} v_{j}\left|G_{i j}^{\dagger}(L+2,0)\right|^{2}
$$

and

$$
\left|r_{i j}\right|^{2}=\left|i \sqrt{v_{i} v_{i}} G_{i j}^{+}(0,0)-\delta_{i j}\right|^{2},
$$

where $G_{i j}^{+}\left(n, n^{\prime}\right)$ is the retarded Green's function with source at $n^{\prime}$ of the $x$ coordinate of channel $j$ and receiver at $n$ of channel $i$. Positions 0 and $L+2$ are located inside the incoming and outgoing sides of the leads, respectively. The channel velocity $v$ in Eqs. (4), (7), and (8) is given by $\partial E / \partial k_{x}$ evaluated at $k_{x}(i)$. Technically, we have assigned zero value to any hopping matrix element connecting to empty site(s). Thus the infinite site energy appeared in Eq. (3) becomes irrelevant in the calculation.

Here we would also like to point out that, in the strongly localized regime, the recursive Green's method used in our calculation of $G_{i j}\left(n, n^{\prime}\right)$ can also be used to probe these wave functions which peak near the center of the disordered medium. In order to probe these func- tions, resonant-tunneling method ${ }^{15}$ have been used to locate the corresponding resonant energies. At each resonant energy, the function $G_{i j}(n, 0)$ is calculated explicitly for every $n$ lying inside the disordered medium, i.e., $1<n<L$. Since the dimensionless conductance at resonant energy is usually of order $1(<<L)$, Eq. (4) can be replaced by

$$
g=\frac{G}{\left(e^{2} / h\right)}=2 \sum_{i=1}^{L_{0}} T_{i}=2 \operatorname{Tr} t t^{+} .
$$

The matrix $t t^{+}$can be diagonalized to obtain eigenvalues $\left\{g_{\alpha}\right\}$ and eigenvectors $\left\{U_{\alpha}(i)\right\}$, with $\alpha=1,2, \ldots, L_{0}$. At resonant energies, most of the $g_{\alpha}$ are practically zero, and only one (or two) eigenvalue(s) is (are) nonzero and contribute to the conductance. These nonzero eigenvalues are always bounded between 0 and 1 . In most cases, there is only one eigenchannel capable of transmitting electrons and this eigenchannel can provide a probe to explore the wave function, which is believed to be nondegenerate. The corresponding wave function of the coordinate $(n, y)$ is then proportional to the Green's function and given by

$$
\phi_{\alpha}(n, y) \propto \sum_{i, j=1}^{L_{0}} w_{i}(y) G_{i j}^{+}(n, 0) U_{\alpha}(j),
$$

where $U_{\alpha}(j)$ is the eigenfunction and $w_{i}(y)$ is the part of the wave function along the $y$ axis of the $i$ th channel in the pure lead. The general property of the matrix $t t^{+}$ and its effective eigenchannels have been discussed in Ref. 16 in connection with universal conductance fluctuations.

\section{MACROSCOPIC TRANSPORT BEHAVIOR}

Calculations based on quantum percolation model as described above have yielded a number of realistic

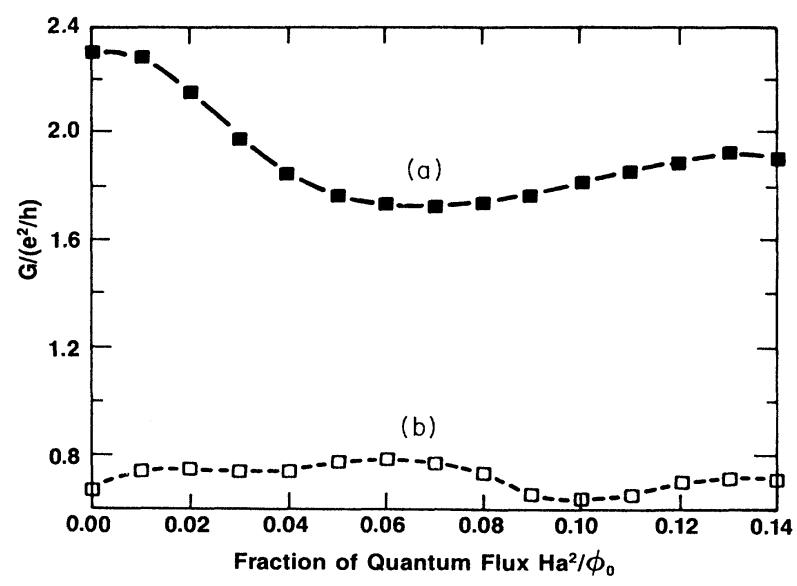

FIG. 1. At $E / T=0.01$ and $p=0.75$, dimensionless conductance $G /\left(e^{2} h\right)$ is plotted as a function of perpendicular magnetic field, expressed in units of fraction of quantum flux $\phi_{0}$. Curve denoted (a) is for sample size $7 \times 7$, averaged over 600 configurations, curve denoted (b) is for sample size $15 \times 15$, averaged over 800 configurations. 


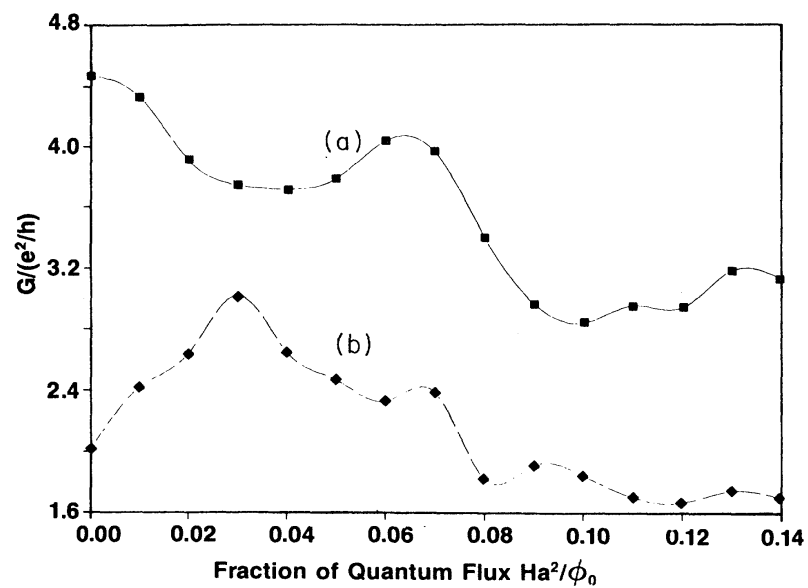

FIG. 2. At $E / T=0.01$ and $p=0.85$, dimensionless conductance $G /\left(e^{2} h\right)$ is plotted as a function of perpendicular magnetic field, expressed in units of fraction of quantum flux $\phi_{0}$. Curve (a) is for sample size $15 \times 15$, curve (b) is for sample size $30 \times 30$. The results are averaged over 200 configurations.

features observed experimentally. Here we show the results of numerical calculations with Fermi energy fixed at $E / t=0.01$, i.e., close to the band center. The dimensionless magnetoconductance calculated with $p=0.75,0.85$, and various sizes $L$ are shown in Figs. 1 and 2, where the fraction of quantum flux, $f=\mathrm{Ha}^{2} / \phi_{0}$, is used as the unit of magnetic field in the horizontal axis. Curves (a) and (b) in Fig. 1 are obtained from the average of 600-800 configurations for a sample of size $7 \times 7(L=7)$ and $15 \times 15(L=15)$, respectively. It is seen that whereas for the smaller sample, corresponding to higher temperatures, the initial variation of the conductance is down-

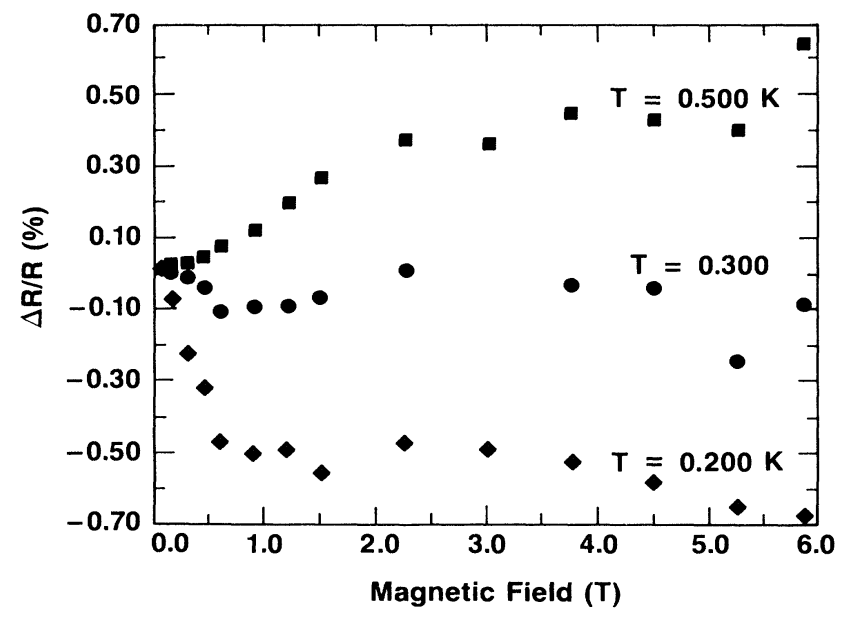

FIG. 3. Measured magnetoresistance, $\Delta R / R_{0}$, plotted as a function of magnetic field for three different temperatures. The sample is a granular $\mathrm{Cu}$ film with metal fraction of $64 \%$. Data is provided by Beamish, Patterson, and Unruh (Ref. 4). ward (positive magnetoresistance), for the larger sample, corresponding to lower temperatures, the opposite is true (negative magnetoresistance). Therefore we have clearly seen a change in the sign of magnetoresistance as the temperature is varied due to the increasing importance of coherent backscattering at lower temperatures. This is indeed seen experimentally as shown in Fig. 3 for granular $\mathrm{Cu}$ films ${ }^{4}$ with metal fraction of $64 \%$.

This sign change can be viewed as the result of the competition between two terms shown on the right-hand side of Eq. (1) for the weak-scattering limit. The first term always gives normal metallic conductance while the second term gives the coherent backscattering contribution. As the value of $p$ is increased, the increase of elastic mean free path $l$ is expected to push the sign change in magnetoresistance to larger sample sizes (and hence lower temperatures). This is indeed what has been seen in our numerical calculations. For the case of $p=0.85$, Fig. 2 shows that the sign change occurs for sizes between 16 and 31 . It should be noted here that we choose $E / t=0.01$ because the states are more localized in the band center for this quantum percolation model ${ }^{17,18}$ (see also Fig. 6). No qualitative difference is expected if one moves away from the band center.

Another common feature for both theory (Figs. 1 and 2) and experiment (Fig. 3) is the oscillating nature of the magnetoconductance as magnetic field varies. This is due to the existence of a length scale, defined by the granular particle size, which can interfere with the magnetic length $L_{H}+\sqrt{h c / 4 e H}$. The origin of the oscillation may be made transparent by examining the conductance of an ordered sample in a magnetic field which is shown in Fig. 4 for a sample of size $L=63$. The structure seen in the magnetoconductance is found to be directly related to the variations in the $2 \mathrm{D}$ ("butterfly") spectrum found by Hofstadter. ${ }^{19}$ For instance, the positions of the peaks and dips shown in Fig. 4 are exactly the values which give the commensurate fluxoid states. Thus the oscillations seen in Figs. 1 and 2 are the remnant effect of commensurate

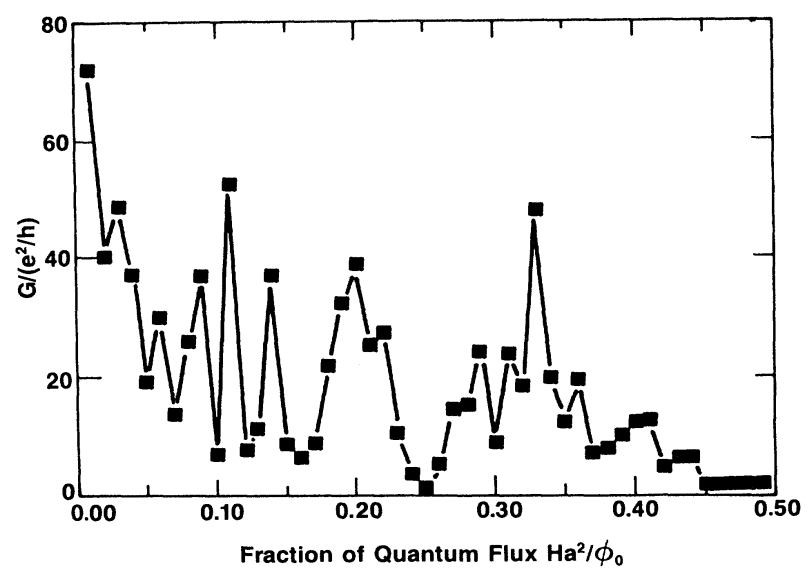

FIG. 4. Dimensionless conductance $G /\left(e^{2} / h\right)$ is plotted as a function of magnetic field in units of fraction of $\phi_{0}$ for a $p=1$ sample of size $63 \times 63$. 


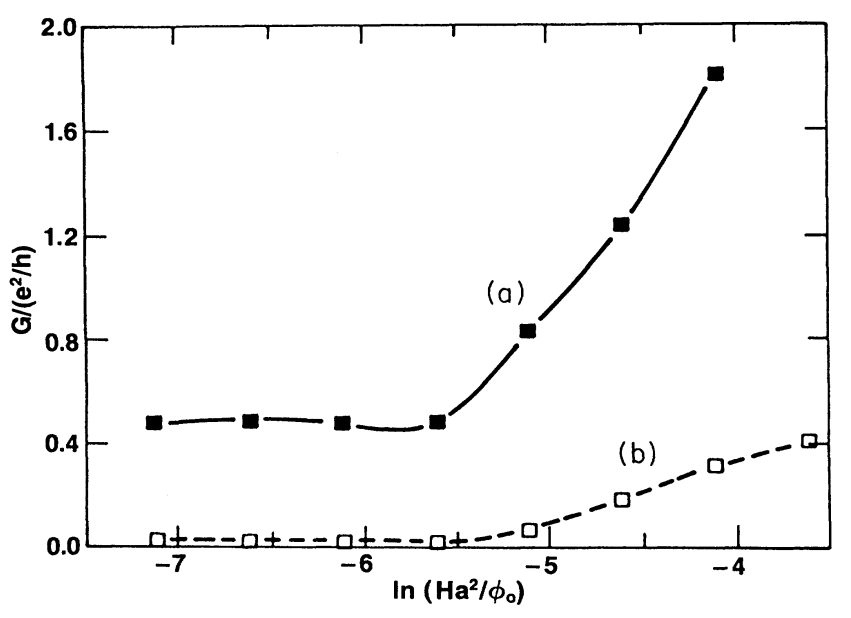

FIG. 5. For $E / t=0.01$ and a fixed sample size $60 \times 60$, dimensionless conductance $G /\left(e^{2} / h\right)$, averaged over 100 configurations, is plotted as the logarithm of $f$. Curve (a) is for $p=0.85$, the slope is 0.90 ; curve (b) is for $p=0.80$, the slope is 0.24 .

fluxoid state after configurational averaging. In order to see the relevance of our calculations to the experimental data, we can choose an average metal particle size to be $a=100 \AA$. A flux fraction of $f=0.14$ shown in Figs. 1 and 2 corresponds to about 7 Telsa which is just the scale of field shown in Fig. 3. The oscillation amplitude is expected to be very much reduced for the experimental data due to the particle size distribution and the finite thickness of the sample.

Logarithmic dependence of the conductance is generally expected for 2D samples at low field and in the weakscattering regime as shown in Eq. (2). However, the coefficient of the logarithmic dependence is by no means universal. In fact, it is strongly dependent on the scattering strength. In Fig. 5 the averaged (over 100 configurations) dimensionless conductance $g\left[=G /\left(e^{2} /\right.\right.$ $h)$ ] is plotted against $\ln (f)$ for the sample size of $L=60$. There are clearly regions of linear behavior in both curves (a) and (b). However, the slopes have the values of 0.90 and 0.24 , respectively. Thus the effect of stronger disorder is seen to reduce the coefficient by a factor of more than 3 from its weak-scattering value. However, it should be noted that the value of 0.90 here for the weaker scattering case differs from $1 / \pi$ predicted by Eq. (2). This could be due to the inaccuracy of the Landauer formula in the weak-scattering limit.

\section{MESOSCOPIC TRANSPORT BEHAVIOR}

\section{A. Universal conductance fluctuations (UCF)}

Recently considerable theoretical and experimental interest has been focused on the study of non-self-averaging quantum transport properties in sample of mesoscopic size. ${ }^{8}$ In such systems, the size $L$ is smaller than the de- phasing length $L_{\phi}$ across which electron loses its phase memory. $L_{\phi}$ is usually determined by the inelastic scattering length. It has been shown from diagrammatic analysis in the weak-scattering limit that, in the regime where $L$ is much greater than the elastic scattering length $L$ but smaller than the localization length, the dimensionless conductance $g=G /\left(e^{2} / h\right)$ fluctuates from sample to sample, and has a universal root-mean-square (rms) fluctuation magnitude of order one. This fluctuation magnitude is independent of degree of disorder, sample size $L$, chemical potential, and magnetic field, provided that the temperature is low enough so that both conditions $L_{\phi}>L$ and $k_{B T}<h D / L^{2}$ are satisfied. Here $D$ is the diffusion constant. ${ }^{8,20-22}$ Numerical tests of UCF have been carried out on various models by using different methods. ${ }^{14,23-25}$ The distribution of mesoscopic conductance has also been studied by invoking random-matrix theory both in the metallic and strongly localized regimes. ${ }^{16,26-29}$ Here we investigate systematically the regime of validity for UCF in the quantum percolation model as a function of percolation probability $p$, sample size $L$, and Fermi energy $E / t$. Taking the ergodic hypothesis, which is believed to hold, we equate the fluctuations of $g$ from sample to sample to that of a given sample as a function of $E / t$ and magnetic field. Although the results presented here are based on calculations using Eq. (4), we have also calculated $g$ by using Eq. (9). No significant differences have been found when the value of $g$ is an order of magnitude smaller than the total number of allowed channels.

In Fig. 6 we plot the dimensionless conductance $g$ as a function of Fermi energy $E / t$ at $p=0.7,0.8$ after averaging over 100 configurations. Due to the symmetry between $\pm E$, only the portion $E / t>0$ is shown. The small value of $g$ near the band center is known to be caused by the existence of a large number of localized states at the

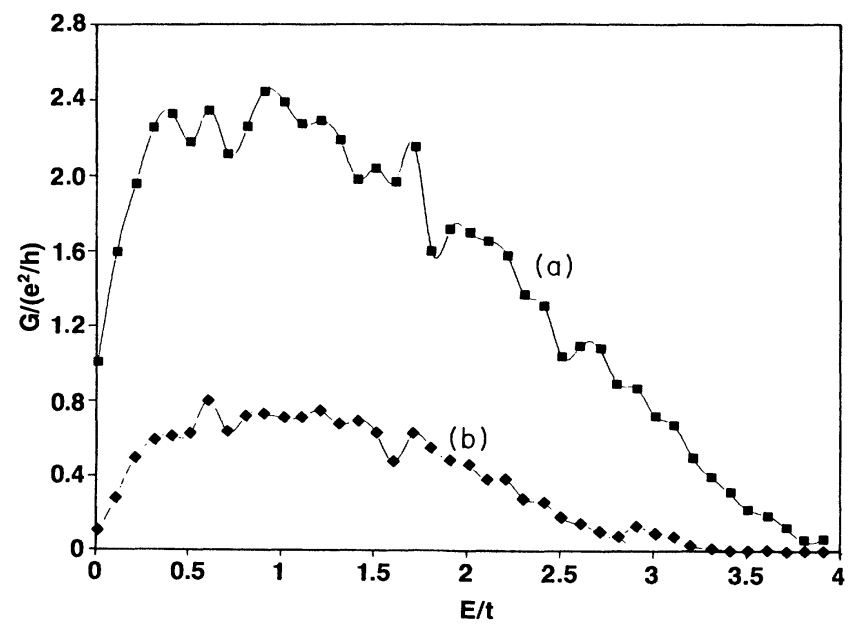

FIG. 6. Dimensionless conductance $G /\left(e^{2} / h\right)$ plotted as a function of Fermi energy $E / t$ for sample size $15 \times 15$. The results are averaged over 100 configurations. Curve (a) is for $p=0.85$, curve (b) is for $p=0.75$. 
band center. ${ }^{17}$ Here we can roughly divide the energy space into two regions: (1) a less localized (higher conductance) region with $0.3<E / t<2.0$ and (2) a more localized (lower conductance) region with $E / t$ outside the range of (1). The region of validity for UCF in these two regions is discussed separately below.

At $E / t=0.5$, the rms value of $\Delta g=\sqrt{\left\langle g^{2}\right\rangle-\langle g\rangle^{2}}$ is plotted in Fig. 7 as a function of sample size $L$ for different values of $p$ ranging from 0.65 to 0.90 . One hundred to six thousands configurations have been generated for each $L$ and $p$ combination. Those configurations which do not percolate across the sample are dropped. In general, less configurations are required for good statistics as $L$ becomes large and $p$ approaches one. Although $\Delta g$ is in general a smoothly decaying function of $L$, there exists a region where the value of $\Delta g$ decays very slowly even for large $L$, e.g., $0.75<p<0.85$ and $L>30$. The value of $\Delta g$ in this region agrees well with the theoretical UCF value of 0.862 in $2 \mathrm{D}$. For $p$ close to one, a much larger $L$ is required to reach the region of UCF since the elastic scattering length increases. On the other hand, as $p$ becomes closer to the percolation threshold $p_{c}(=0.593)$, the localization length becomes smaller and the region of UCF is also limited to small $L$. Both of these features can be seen clearly in curves of $p=0.9$ and 0.7 in Fig. 7. It is worthwhile to point out that in the region of UCF the average $g$ is always an order of magnitude smaller than the total number of allowed channels. From the analysis of a number of effective channels, ${ }^{16}$ this implies the sample size to be at least an order of mag-

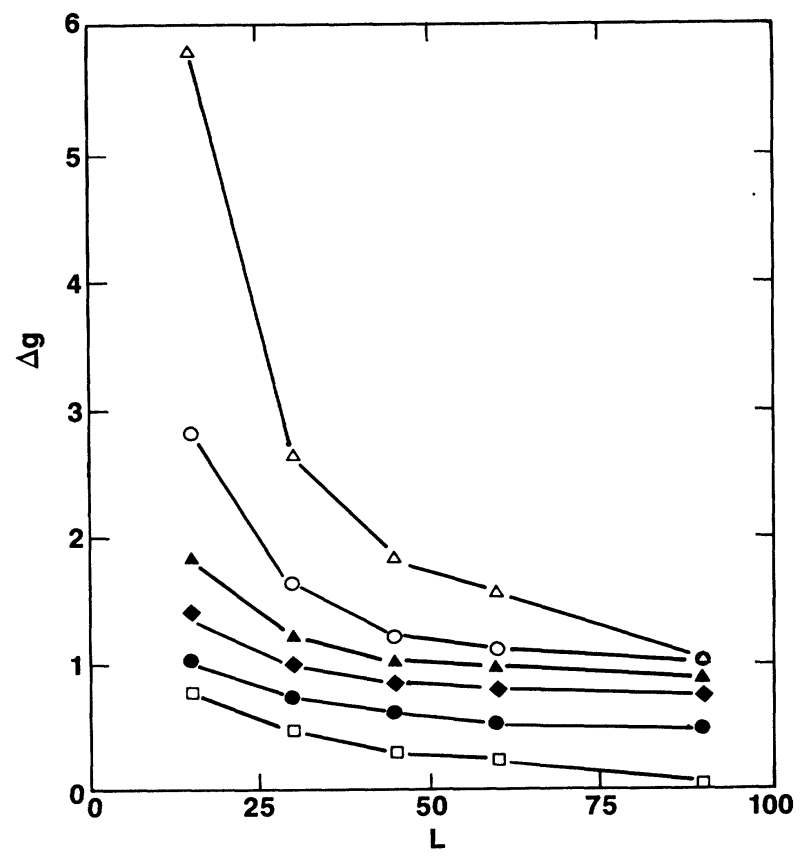

FIG. 7. The rms dimensionless conductance fluctuation $\Delta g$ plotted function of sample size $L$ for various values of $p: \Delta$ for $p=0.90, \bigcirc$ for $0.85, \Delta$ for $0.80, \diamond$ for $0.75, \diamond$ for 0.70 , and $\square$ for 0.65 . The value of $E / t$ is 0.5 .

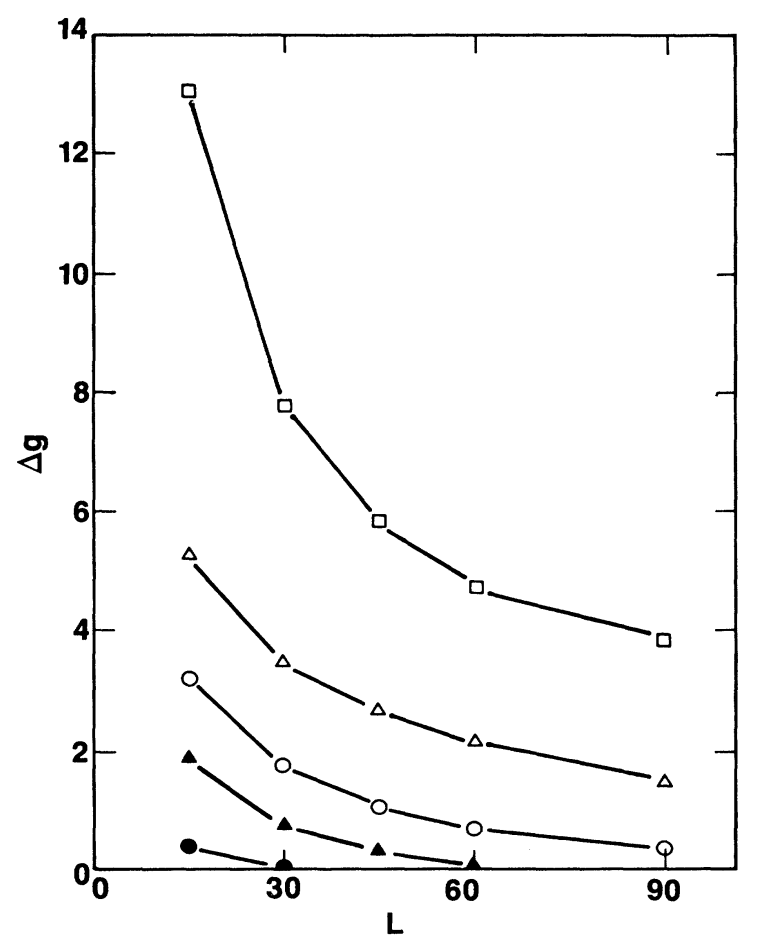

FIG. 8. The rms dimensionless conductance fluctuation $\Delta g$ plotted as a function of sample size $L$ for various values of $p$ : $\square$ for $p=0.95, \triangle$ for 0.90 , $\bigcirc$ for $0.85, \Delta$ for 0.80 , and $\bullet$ for 0.75 . The value of $E / t$ is 0.01 .

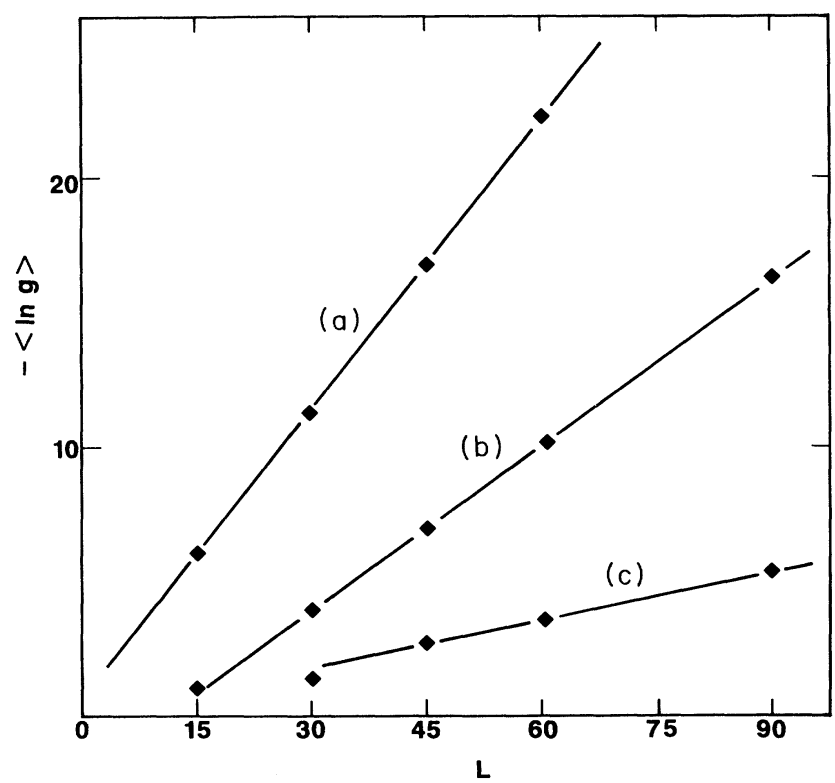

FIG. 9. The averaged value of $-\operatorname{lng}$ plotted as a function sample size $L$. The data points show a linear behavior. The inverse of the slope gives the localization length. (a) is for $p=0.75$, (b) is for $p=0.80$, and (c) is for $p=0.85$. The value of $E / t$ is 0.01 . 
nitude larger than the elastic scattering length, i.e., $10 l<L$. In this case, Eq. (4) can be replaced by Eq. (9), which is the basis of many theoretical discussions on UCF.

The situation is quite different in the more localized region, $0<E / t<0.3$. At $E / t=0.01$, the curves of $\Delta g$ for different values of $p$ are shown in Fig. 8. There is hardly a region of constant $\Delta g$ that can be identified. This may be understood as follows. For $p<0.85$ the localization length can be estimated by using the finite-size scaling method. In Fig. 9, the average of the logarithmic conductance is plotted as a function of $L$. The linear behavior signals the exponential decay of the conductance. From the relation $\xi_{\text {loc }}=-d L / d\langle\ln (g)\rangle$, we obtain the localization lengths $\xi_{\text {loc }}$ for $p=0.75,0.80$, and 0.85 to be $2.7,5.0$, and 16.4 , respectively. These numbers are small compared to our size of interest, i.e., $L=30$ to 90 . When $p$ becomes greater than 0.85 , there is a drastic increase in the localization length. The corresponding increase in the elastic scattering length, which is proportional to $(1-p)^{-1}$ in the weak disorder limit, pushes the region of UCF to much larger sample sizes $(L>90)$. A rough estimate of elastic scattering length can be made by using the number of effective channels, ${ }^{16}$ which is the dimensionless conductance evaluated by using Eq. (9). In the extended region ( $L<$ localization length) we have $g=N_{\text {eff }}$ $=2 L_{0} l / L$ where $L_{0}$ is the number of allowed channels. From this relation, the values of $l$ are found to be about $l=12,4$, and 1.6 for $p=0.95,0.9$, and 0.85 , respectively. Thus it requires at least $L>120(=10 l)$ in order to see UCF for $p=0.95$. This is consistent with the corresponding curves shown in Fig. 8.

If the same considerations are now applied to the cases shown in Fig. 7, we found that the values of $l$ at $E / t=0.5$ are about $7,3.7,2$, and 1.3 for $p=0.90,0.85$, 0.80 , and 0.75 , respectively. Although these numbers are about a factor of 2 higher than the corresponding numbers in the case of $E / t=0.01$, the localization length $\xi_{\text {loc }}$ is also much greater. From finite-size scaling, localization length is found to be $\cong 30$ for $p=0.70$. A rough estimate gives $\xi_{\text {loc }} \cong 100$ and 250 for $p=0.75$ and 0.80 . This is again consistent with the corresponding curves shown in Fig. 7. Thus we expect the curve for $p=0.8$ to remain flat up to $L=200$.

Overall, our analysis confirms the theoretical prediction that the UCF exists in regions where sample size $L$ is much greater than the elastic scattering length $l$ but smaller than the localization length $\xi_{\text {loc }}$, independent of disorder and Fermi energy.

\section{B. Statistical distribution of $g$}

Here we would like to discuss the statistical distribution of the dimensionless conductance $g$. Although there is a general belief that the distribution of $g$ should be normal in the extended side while log-normal in the localized side $; 6,16$ yet, to our knowledge, there exists no systematic numerical study of this problem. Extensive numerical calculations in this work have revealed that in general, there are three types of distribution in the following three regimes: (1) extended limit, (2) localized limit, and (3) in-

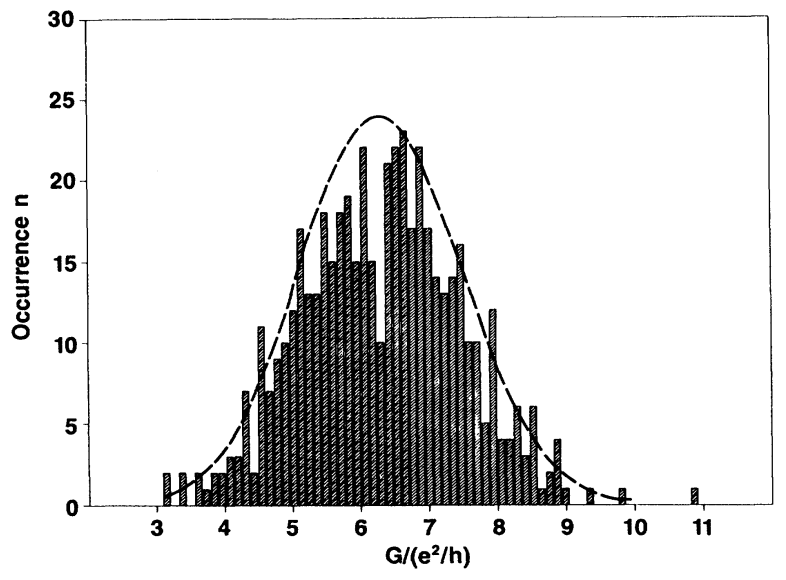

FIG. 10. Histogram of the conductance distribution for 500 configurations. The parameter values are $E / t=0.01, p=0.95$, and $L=60$. The dashed line is a Gaussian line shape to guide the eye.

termediate regime. In the extended limit, transport is ballistic, or nearly so. The sample size $L$ is considered to be larger (but not much larger) than the elastic scattering length $l$ but much smaller than the localization length $\xi_{\text {loc }}$, i.e., $l \leq L<<\xi_{\text {loc }}$. The distribution of $g$ in this limit is found to be normal. In terms of $g$ and $\Delta g$, the corresponding criterion for normal distribution is that $g \gtrsim 3 \Delta g$. In the localized limit, the sample size is considered to be much greater than localization length, i.e., $\xi_{\text {loc }}<L$. The corresponding distribution then becomes log-normal. The intermediate regime lies between the above two limits and the distribution of $g$ is neither nor-

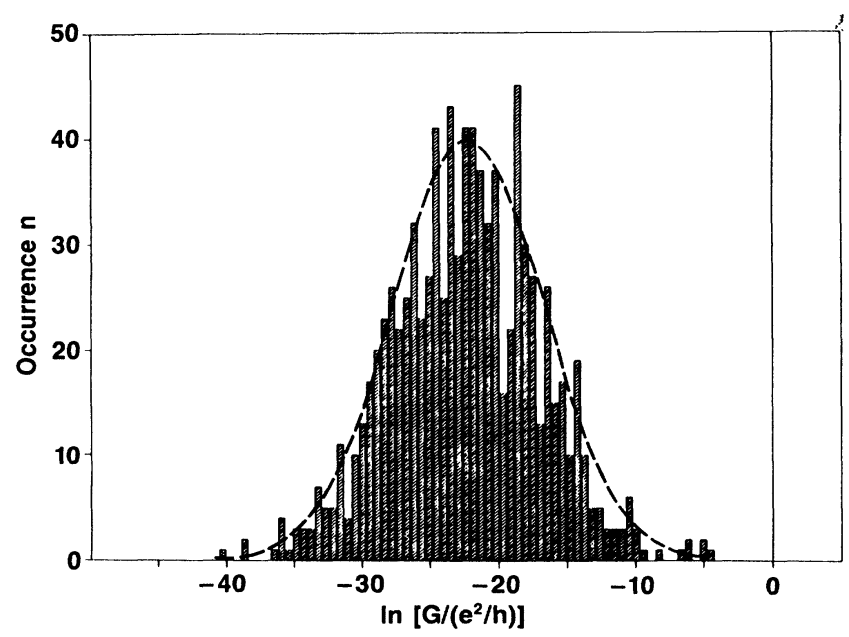

FIG. 11. Histogram of the conductance distribution plotted as a function of $\ln \left[G /\left(e^{2} / h\right)\right]$ for 900 configurations. The parameter values are $E / t=0.01, p=0.75$, and $L=60$. The dashed line is a Gaussian line shape to guide the eye. 
mal nor log-normal.

Three typical cases are shown in Figs. 10-12 for $L=60, E / t=0.01$ and $p=0.95,0.85$, and 0.75. At $p=0.95$, the value of $l$ is about 12 from the previous discussion and the localization length is expected to be much greater than 60. The distribution of $g$ for 500 configurations is shown in Fig. 10 and is seen to be a very good Gaussian. At $p=0.75$, the localization length is only 2.7 (much smaller than sample size $L=60$ ), and the histogram of $\ln (g)$ is shown in Fig. 11 for 900 configurations. The distribution is found to be a good Gaussian in terms of $\ln (g)$, i.e., the distribution of $g$ is a log-normal in this regime. However, the case of $p=0.85$ belongs to the intermediate regime with $\xi_{\text {loc }}=16$, which is not much smaller than the sample size (60). The distribution, with 700 configurations, is shown in Fig. 12 in terms of both $g$ and $\ln (g)$. They are clearly nonGaussian. This peculiar distribution can be viewed as the intermediate one between normal and log-normal, i.e., the large conductance part is normal [Fig. 12(a)] while the smaller conductance part is log-normal [Fig. 12(b)].
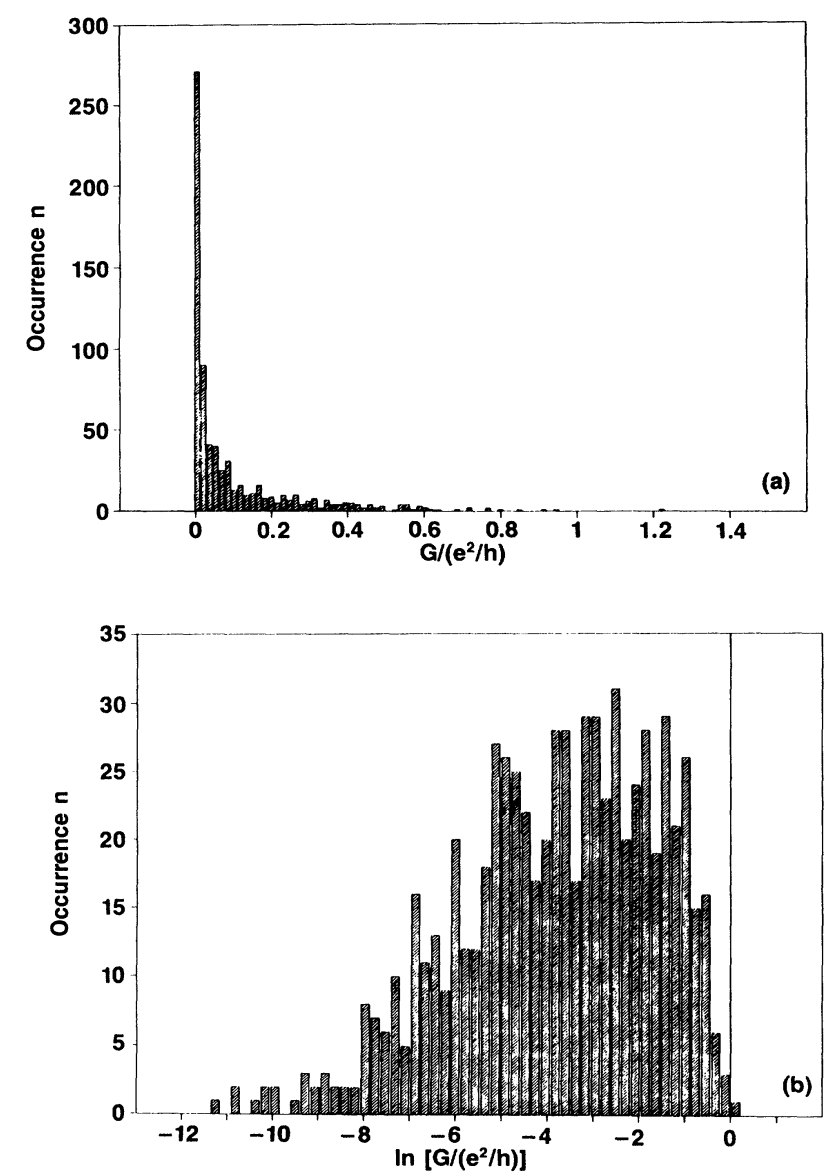

FIG. 12. (a) Histogram of conductance distribution for 700 configurations. The parameter values are $E / t=0.01, p=0.85$, and $L=60$. (b) Histogram of the conductance distribution plotted as a function of $\ln \left[G /\left(e^{2} / h\right)\right]$ for 700 configurations. The parameter values are the same as $(a)$.
In fact, the region of UCF $\left(10 l<L<\xi_{\text {loc }}\right)$ lies in this intermediate regime. In Fig. 13 we show the different transport regimes of the quantum percolation model as delineated by $p$ and sample size $L$.

\section{Behavior at the percolation threshold}

As the value of $p$ approaches the percolation threshold $p_{c}$, electrons are strongly localized for all energies $E / t$. Unlike the corresponding regime in the Anderson model, in our model there exists a geometrical correlation length $\xi_{p}$ which characterizes the density fluctuation of a percolation cluster. Within the scale $\xi_{p}$ the system behaves as a fractal, and beyond $\xi_{p}$ the system is homogeneous. ${ }^{30}$
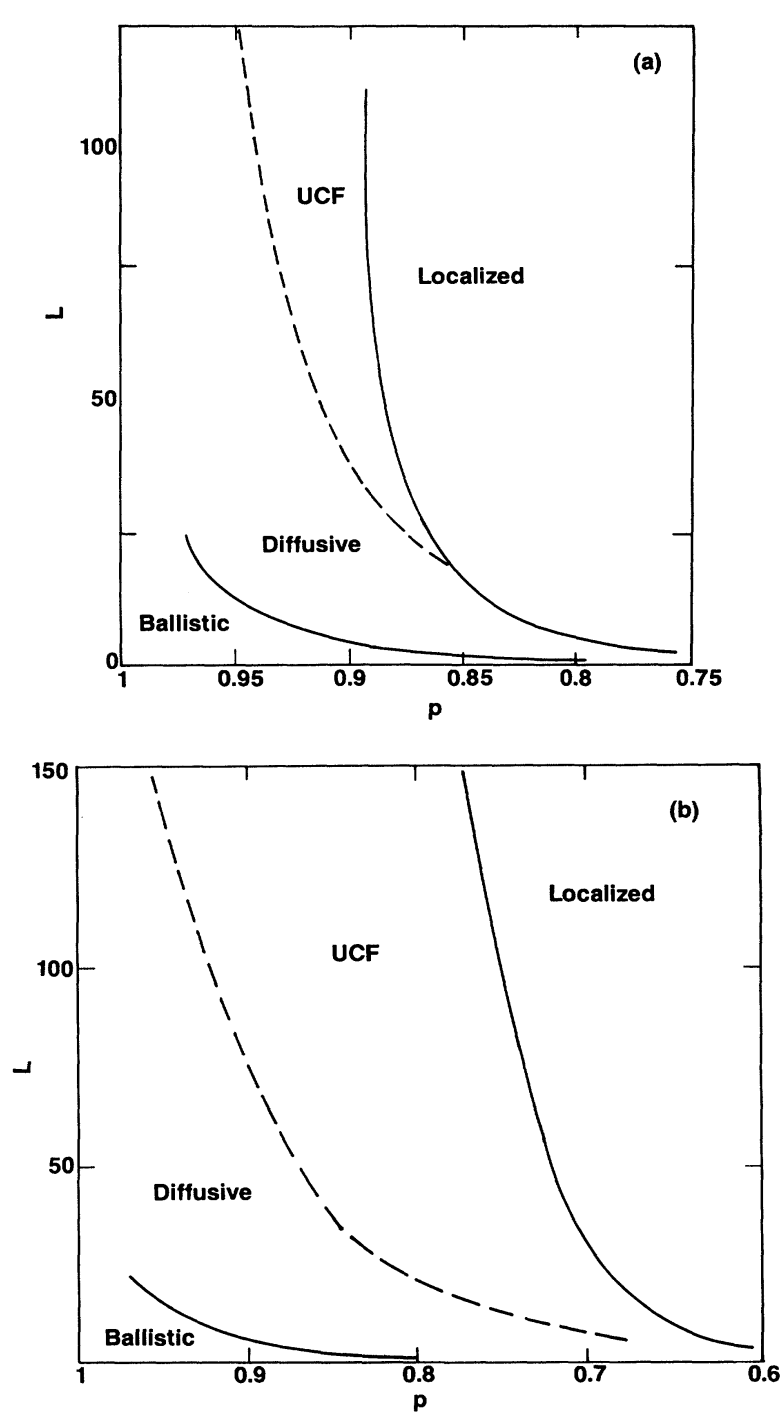

FIG. 13. Transport regimes of the $2 \mathrm{D}$ quantum percolation model as a function of $L$ and $p$. The upper solid line denotes the localization length, the lower solid line denotes the mean free path. Dashed line denotes ten times the mean free path. (a) $E / t=0.01$, (b) $E / t=0.5$. 
At $p_{c}(=0.593)$, the whole system becomes a fractal with fractal dimension $\sim 1.89$. Here, we would like to study the statistics of $\ln (g)$ and the localization behavior of the wave function with such underlying fractal geometry. In particular, we examine the evidence for superlocalization, i.e., electronic wave function decays faster than simple exponential.

In this strongly localized regime, there are at least two methods which can be used to study the localization behavior of the wave function. One is the finite-size scaling method used previously. It can provide the averaged properties of electron localization. The other is the resonant-tunneling method, ${ }^{15}$ which can provide us with a tool to directly probe the wave function as described in Sec. II. Below we give a brief description of the resonant-tunneling method and our extension of the method.

The problem of resonant tunneling is well understood in $1 \mathrm{D}$. Its generalization to $2 \mathrm{D}$ has recently been carried out for the Anderson model. ${ }^{15}$ However, in that work the measurement of localization length is still indirect, i.e., near the resonant energy the spinless dimensionless conductance $g / 2$ is given by the following relation: ${ }^{15}$

$$
g=\frac{2 \Gamma^{L} \Gamma^{R}}{\left(E-E_{r}\right)^{2}+\left(\frac{\Gamma^{L}}{2}+\frac{\Gamma^{R}}{2}\right)^{2}},
$$

where $E_{r}$ is the energy of the localized state and $\Gamma^{L}, \Gamma^{R}$ are related to the localization length $\xi_{\text {loc }}$ by $\Gamma^{L}$ $\exp \left(-x / \xi_{\text {loc }}\right)$ and $\Gamma_{\infty}^{R} \exp \left[-(L-x) / \xi_{\text {loc }}\right]$. Here $L$ is the sample size and $x$ denotes the position of the localization center along the tunneling direction. For consistency with our previous discussions, here we define $\xi_{\text {loc }}$ as the decay length of the probability density. By measuring the height $\beta$ and width $\gamma$ of the resonant peak, the localization length $\xi_{\text {loc }}$ is obtained as

$$
\xi_{\text {loc }}=L / \ln \left(\beta \gamma^{2}\right) \text {, }
$$

with

$$
\beta=\frac{\Gamma^{L} \Gamma^{R}}{\left[\frac{\Gamma^{L}}{2}+\frac{\Gamma^{R}}{2}\right)^{2}}
$$

and

$$
\gamma=\frac{\Gamma^{L}}{2}+\frac{\Gamma^{R}}{2}
$$

We have applied the above method to our model for $E / t=0.5$. Among many hundred realizations carried out at $p=p_{c}$, we have been able to found some configurations which have resonant peaks near $E / t=0.5$. The rest of the configurations have values of $g$ many orders of magnitude less than one. A typical tunneling result for a sample of size $L=45$ is shown in Fig. 14 where the shape can be well fitted by a Lorentzian with $E_{r}=0.500189, \beta=0.8$, and $\gamma=1.2 \times 10^{-4}$. By using Eq. (12), the localization length is found to be about 2.5. The result of finite-size scaling is also shown in Fig. 15

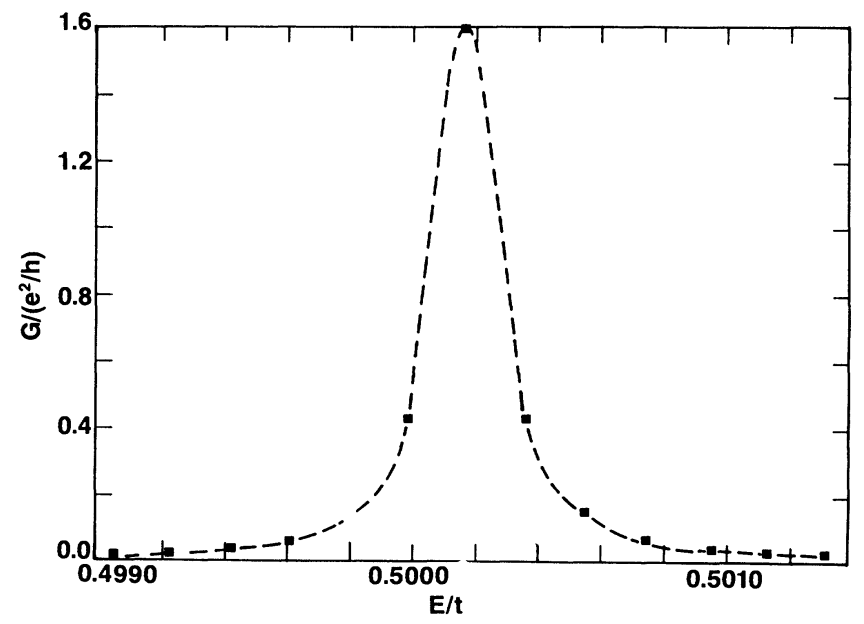

FIG. 14. Resonant peak of a particular configuration of size $L=45$ at the percolation threshold $p_{c}=0.593$.

from which we find $\xi_{\text {loc }}=3.30$. Although the values of $\xi_{\text {loc }}$ obtained by the resonant-tunneling method are consistent with the result of finite-size scaling, one can still not probe directly the shape of each individual wave function.

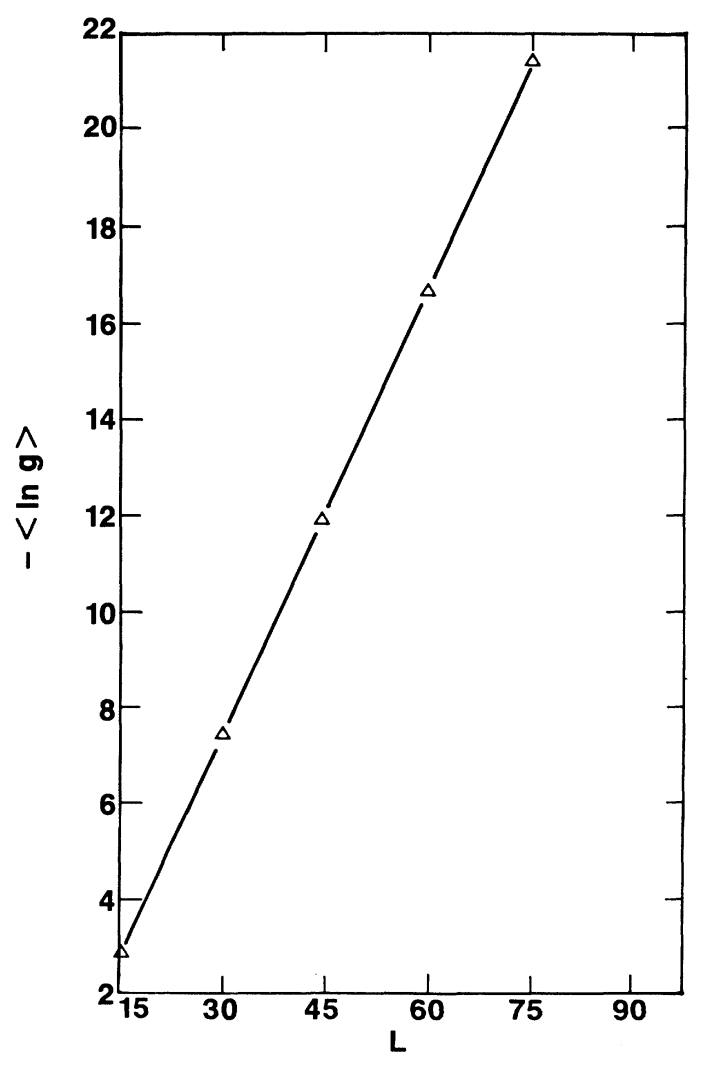

FIG. 15. $-\langle\operatorname{lng}\rangle$ plotted as a function of sample size $L$ for $p=p_{c}$ and $E / t=0.5$. 
Here we extend the resonant-tunneling method to the direct evaluation of wave functions. As described in Sec. II, in the strongly localized regime most eigenvalues of the matrix $t t^{+}$are vanishingly small and usually only one (or two) eigenvalue(s) is (are) on the order of one. By transferring the problem into an eigenchannel representation, the explicit form of the wave function can be obtained via Eq. (10) by evaluating the Green's function with resonant eigenchannel as a source. Since one of our aims is to investigate the possibility of a superlocalization on a fractal geometry, ${ }^{31,32}$ we have calculated the wave functions at those resonant energies found previously at $p_{c}$ and $E / t \simeq 0.5$. We first locate the center of a wave function where the probability density is maximum, then the average probability density as a function of distance $R$ from the center is calculated. A typical result is shown in Fig. 16 where the logarithm of the average probability density $I(R)$ is plotted as a function of $R$. This corresponds to the case of Fig. 14 discussed above. The center of the wave function is at $x=24$ and $y=34$, which lies just near the midplane of the sample $(L=45)$. The data in Fig. 16 can be fitted by a linear line. From the slope we found the localization length to be 2.5 , agreeing well with the estimate of indirect tunneling method [Eq. (12)]. Although Fig. 14 shows an exponential decay of the wave function, we prefer to answer the question of superlocalization by using the following statistical analysis. Eight resonant cases have been chosen with sizes ranging from $L=46$ to 73 and energies ranging from $E / t=0.2$ to 0.5 . Each of these data can be fitted by a linear line. The deviations of these data points from the best linear fits are superimposed on a single data sheet where the distance $R$ in the horizontal axis is renormalized by the localization length found for each case. The result is shown in Fig. 17 where different symbols denote different cases. Since all the data points in Fig. 17 distribute evenly above and below the line of zero fluctuation, there is no statistical indication of superlocalization. If there is any superlocalization, one would expect the data points to exhibit sys-

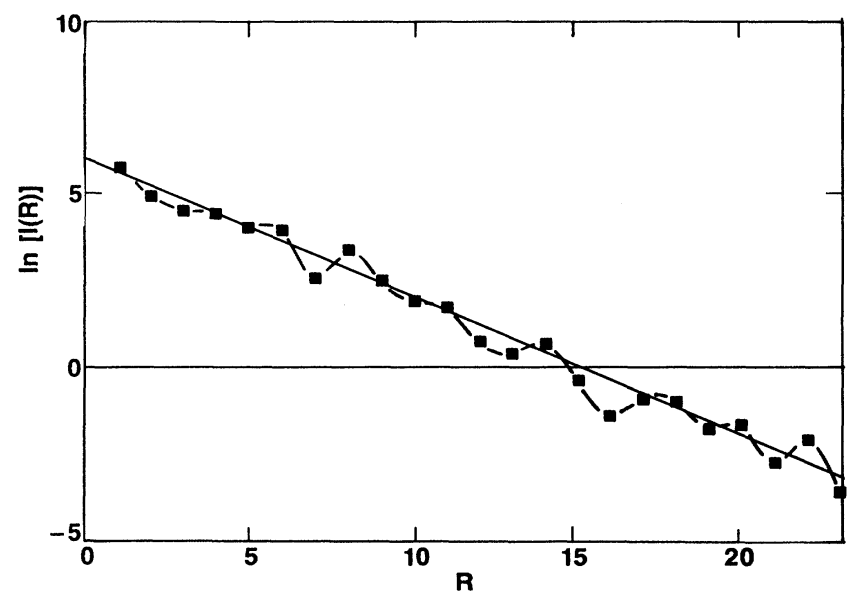

FIG. 16. Logarithm of averaged probability density $I$ plotted as a function of distance $R$ from the center of the localized wave function obtained from the resonant peak shown in Fig. 14.

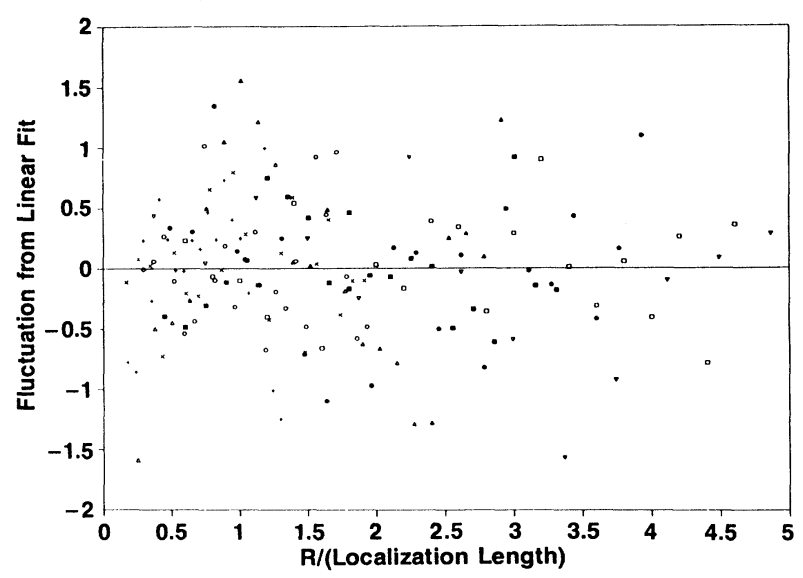

FIG. 17. Statistical deviations of averaged probability density $I(R)$ from the linear behavior for eight different wave functions obtained from the resonant-tunneling method. The distance $R$ from each localization center is normalized by the corresponding localization length. The distribution is seen to be uniform and symmetric about zero.

tematic deviation from a uniform distribution. Our conclusion is therefore consistent with the results of a recent work using direct numerical simulations, ${ }^{32}$ i.e., there is no superlocalization.

Besides the single-resonant-peak cases, there are also cases where two-resonant peaks overlap when the energy separation of two localized states is less than their widths. A typical example is shown in Fig. 18 for a sample of $L=60$. The resonant energies are $E_{r} / t=0.5000$ and 0.5014 , respectively. Although this double-peak case has been discussed before, ${ }^{15}$ its wave functions have not been explored. From the calculation of the wave function at each resonant energy, we found that these two wave functions are centered approximately at an equal distance

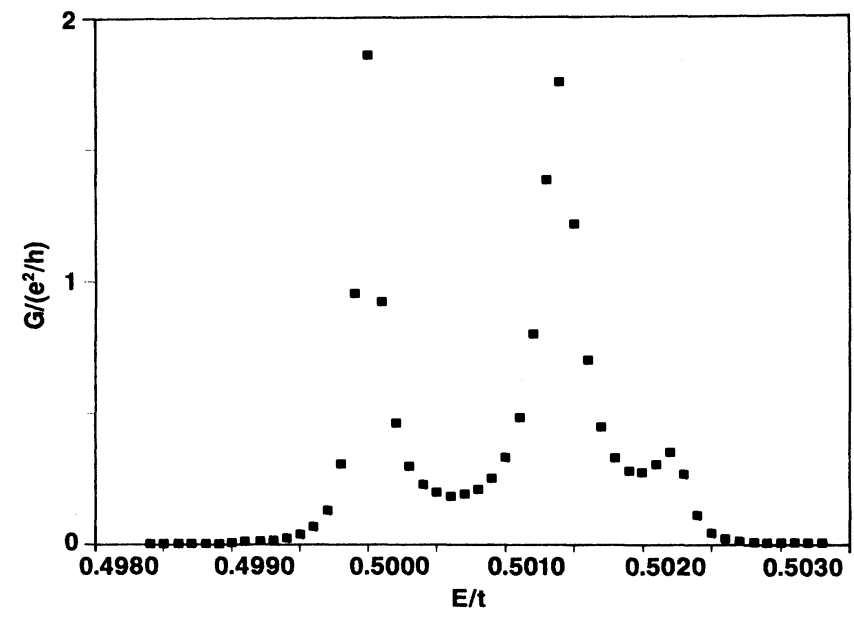

FIG. 18. Two overlapping resonant peaks of a particular configuration of size $L=60$ at the percolation threshold. 


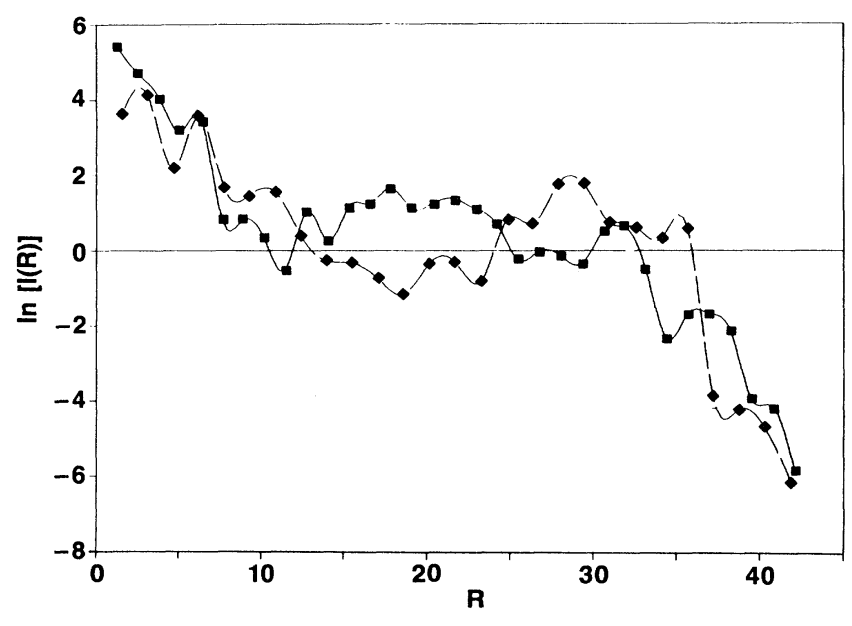

FIG. 19. Logarithm of averaged probability density plotted as a function of distance $R$ from the centers of the two localized wave functions obtained from the two resonant peaks shown in Fig. 18.

away from the midplane of the sample. The probability density decays from one center and picks up a small resonant peak in the neighborhood of the other center due to the closeness in energy of the two states. For the resonant case of Fig. 18, the centers are at $x=23, y=35$ for $E_{r} / t=0.5000$, and $x=39, y=12$ for $E_{r} / t=0.5014$, respectively. The logarithm of each probability density is plotted in Fig. 19 as a function of $R$. Both curves show a small bump in the neighborhood of $R=21$, which is the distance between two centers.

The effects of the magnetic field on the localization properties have also been studied. Again eight singleresonant cases have been analyzed, and the wave function is found again to decay exponentially. There is no sign of superlocalization even in the presence of a magnetic field. The change of localization length under a strong magnetic field (for a single sample) has been studied in detail for the Anderson model. ${ }^{15}$ Similar behavior is expected in the present quantum percolation model. Here we are more interested in the behavior of averaged localization length in the low field region. The averaged localization length at low fields is studied by using the finite-size scaling method. For each sample size ranging from 15 to 60 , hundreds of percolation configurations were averaged. In Fig. $20 \xi_{\text {loc }}$ is plotted as a function of $f\left(-\mathrm{Ha}^{2} / \phi_{0}\right)$. The general trend of $\xi_{\text {loc }}$ variation under a magnetic field is consistent with the known results. ${ }^{13,15,33}$ However, the data shown in Fig. 20 seem to indicate a linear dependence in the low-field region rather than a square-root dependence obtained in the hopping model. ${ }^{33}$

\section{CONCLUDING REMARKS}

A quantum percolation model has been introduced to study both the macroscopic and mesoscopic transport properties of granular metal films. This model takes into account the granular properties of the film and enables us to study the magnetoconductance in the strong scattering and strong field regimes. A multichannel Landauer for-

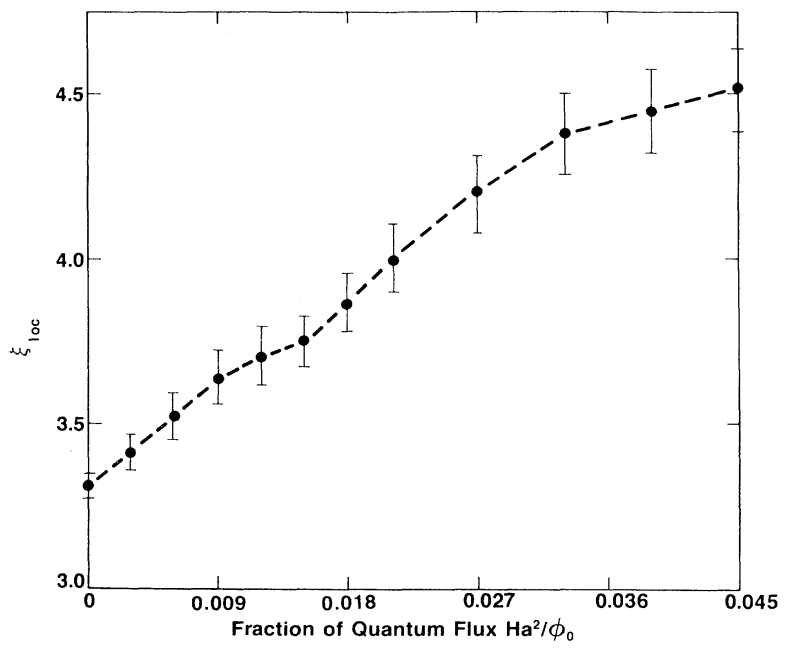

FIG. 20. The localization length at $p=p_{c}$ and $E / t=0.5$ plotted as a function of the magnetic field, expressed in units of fraction $f$ of the quantum flux $\phi_{0}$. Error bars are shown.

mula is used to calculate the conductance. The averaged magnetoconductance calculated shows a sign change as the sample size varies. There also exists oscillations in the magnetoconductance in large fields due to the finite granular particle size. Both observations are consistent with some experimental data.

The mesoscopic properties of this model have been thoroughly studied. On the extended side, the rms of conductance fluctuations are calculated for various values of percolation probability $p$, sample size $L$, and Fermienergy $E / t$. A region of universal conductance fluctuations is identified which is consistent with the known UCF criterion, i.e., elastic scattering length $10 l \lesssim L<10-$ calization length $\xi_{\text {loc }}$. The distribution of conductance is normal in the extended limit ( $\left.l \leq L \leq \xi_{\text {loc }}\right)$ and log-normal in the localized limit $\left(\xi_{\text {loc }}<<L\right)$. Between these two limits, the distribution is of mixed type.

The behavior of the wave function in the strongly localized regime is studied by both the finite-size-scaling method for the averaged property and the resonanttunneling method for single-sample properties. The resonant-tunneling method has been extended to explicitly calculate the localized wave function. At percolation threshold $p_{c}$, both methods show the wave function to be exponentially localized. We find no indication of superlocalization. This is also true in the presence of the magnetic field. Our finite-size-scaling results show that averaged localization length is an increasing function of magnetic field and shows a strong linear dependence at low fields.

Although our calculations here are only focused in 2D system, it is straightforward to generalize this model to films with finite thickness or $3 \mathrm{D}$ systems. The study of these problems will be our future endeavor.

\section{ACKNOWLEDGMENTS}

We wish to thank J. Beamish and K. Unruh for many helpful discussions in regard to their data. This work is supported by ONR Contract No. N00014-88-K-003. 
*Also at Bartol Research Institute, University of Delaware, Newark, DE 19716, and Institute of Physics, Academia Sinica, Beijing, People's Republic of China.

${ }^{1}$ See Physical Phenomena in Granular Materials, Materials Research Society Symposium Proceedings, Vol. 195, edited by G. D. Cody, T. H. Geballe, and P. Sheng (MRS, Pittsburgh, Pennsylvania, 1990), and references therein.

${ }^{2}$ P. Sheng, B. Abeles, and Y. Arie, Phys. Rev. Lett. 31, 44 (1973).

${ }^{3}$ P. Sheng and J. Klafter, Phys. Rev. B 27, 2583 (1983).

${ }^{4}$ J. R. Beamish, B. M. Patterson, and K. M. Unruh, in Physical Phenomena in Granular Materials (Ref. 1), p. 129.

${ }^{5}$ G. Bergmann, Phys. Rep. 107, 1 (1984).

${ }^{6}$ P. A. Lee and T. V. Ramakrishnan, Rev. Mod. Phys. 51, 287 (1985).

${ }^{7}$ M. P. van Albada and A. Lagendijk, Phys. Rev. Lett. 55, 2692 (1985).

${ }^{8}$ See, for example, P. A. Lee, A. D. Stone, and H. Fukuyma, Phys. Rev. B 35, 1039 (1987).

${ }^{9}$ M. Büttiker, Y. Imry, R. Landauer, and S. Pinhas, Phys. Rev. B 31, 6207 (1985).

${ }^{10}$ Y. Shapir, A. Aharony, and A. B. Harris, Phys. Rev. Lett. 49, 486 (1982).

${ }^{11}$ Y. Meir, A. Aharony, and A. B. Harris, Phys. Rev. Lett. 56, 976 (1986).

${ }^{12}$ R. E. Peierls, Z. Phys. 80, 763 (1933).

${ }^{13}$ P. A. Lee and D. S. Fisher, Phys. Rev. Lett. 47, 882 (1981).

${ }^{14}$ A. D. Stone, Phys. Rev. Lett. 54, 2692 (1985).
${ }^{15}$ W. Xue and P. A. Lee, Phys. Rev. B 38, 3913 (1988).

${ }^{16}$ Y. Imry, Europhys. Lett. 1, 249 (1986).

${ }^{17}$ S. Kirkpatrick and T. P. Eggarte, Phys. Rev. B 36, 3598 (1987).

${ }^{18}$ C. M. Soukoulis, E. N. Economou, and G. S. Grest, Phys. Rev. B 36, 8649 (1987).

${ }^{19}$ D. R. Hofstadter, Phys. Rev. B 14, 2239 (1976).

${ }^{20}$ P. A. Lee and A. D. Stone, Phys. Rev. Lett. 55, 1622 (1985).

${ }^{21}$ B. L. Alt'shuler, Pis'ma, Zh. Eksp. Teor. Fiz. 51, 530 (1985) [JETP Lett. 41, 648 (1985)].

${ }^{22}$ P. A. Lee, Physica 140A, 169 (1986).

${ }^{23}$ N. Giordano, Phys. Rev. B 36, 4190 (1987).

${ }^{24}$ X. C. Xie and S. Das Sarma, Phys. Rev. B 38, 3529 (1988).

${ }^{25}$ R. Harris and A. Houari, Phys. Rev. B 41, 5487 (1990).

${ }^{26}$ B. L. Al'tshuler and B. I. Shklovskil, Zh. Eksp. Teor. Fiz. 91, 3 (1986) [Sov. Phys. JETP 64, 1 (1986)].

${ }^{27}$ K. A. Muttalib, J.-L. Pichard, and A. D. Stone, Phys. Rev. Lett. 59, 2475 (1987).

${ }^{28}$ K. A. Muttalib, Phys. Rev. Lett. 65, 745 (1990).

${ }^{29}$ J.-L. Pichard, M. Sanquer, K. Slevin, and P. Debray, Phys. Rev. Lett. 65, 1812 (1990).

${ }^{30}$ D. Stauffer, Phys. Rep. 54, 1 (1979).

${ }^{31}$ Y. E. Levy and B. Souillard, Europhys. Lett. 4, 233 (1987); A. B. Harris and A. Aharony, ibid. 4, 1355 (1987).

${ }^{32}$ P. de Vries, H. de Raldt, and A. Lagendijk, Phys. Rev. Lett. 62, 2515 (1989).

${ }^{33}$ E. Median, M. Kardar, Y. Shapir, and X. R. Wang, Phys. Rev. Lett. 64, 1816 (1990). 